\title{
Response of ophthalmologists in Israel to the novel coronavirus (2019-nCoV) outbreak
}

\author{
Lauren M. Wasser ${ }^{1}$ (D) $\cdot$ Elishai Assayag ${ }^{1} \cdot$ Maria Tsessler $^{1} \cdot$ Yishay Weill $^{1} \cdot$ Michal Becker-Cohen $^{1} \cdot$ David Zadok $^{1}$
}

Received: 31 March 2020 / Revised: 6 April 2020 / Accepted: 8 April 2020 / Published online: 28 April 2020

(C) Springer-Verlag GmbH Germany, part of Springer Nature 2020

\begin{abstract}
Purpose The coronavirus disease (COVID-19) pandemic has evolved into a formidable healthcare crisis. Ophthalmologists are at daily personal risk of acquiring and transmitting the virus. Implementation of official practical and protective guidelines can be challenging and is often absent. The purpose of this study was to describe the status of ophthalmology practice in Israel, at the early stages of the outbreak.

Methods A 17-item questionnaire was distributed to ophthalmologists practicing in Israel. Data was obtained regarding demographics and clinical and surgical practice during the pandemic.

Results One hundred and sixty-seven ophthalmologists completed the survey from all regions of Israel. The survey was distributed during the early stages of the outbreak. At this time, no official government guidelines were in place. Most respondents reported no reduction of elective clinic visits and surgeries and no utilization of triage questionnaires. COVID-19 guidelines were reportedly promulgated to hospital ophthalmologists but not to community and private physicians. Personal protective equipment (PPE) measures were reportedly utilized; however, many respondents often acquired them individually. A majority of respondents advocated that healthcare institutions limit clinic and surgery services to emergency services.

Conclusion During the critical early stages of the COVID-19 outbreak in Israel, this study emphasizes the delay in development of emergency guidelines, necessary to protect patients and ophthalmologists from this highly transmissible disease.
\end{abstract}

Keywords Pandemic $\cdot$ COVID-19 · Survey

\section{Introduction}

The coronavirus disease (COVID-19) pandemic is an emerging global health concern [1]. This highly

Lauren M. Wasser, Elishai Assayag and Maria Tsessler contributed equally to this work.

Lauren M. Wasser

laurenw@szmc.org.il

1 Department of Ophthalmology, Shaare Zedek Medical Center Affiliated with the Hebrew University - Hadassah School of Medicine Jerusalem, 9103102 Jerusalem, Israel contagious virus, severe acute respiratory syndrome coronavirus 2 (SARS-CoV-2) [2], causes devastating respiratory disease with a variable incubation time of 214 days [3]. The infection rate (R0), estimated between 1.5 and 3.5 [4], as well as evidence of possible transmission by asymptomatic carriers [5] places healthcare professionals at substantial infection risk.

Ophthalmology practice combines high-volume outpatient and community clinics, elective surgeries, and emergency services. It requires prolonged and close contact with patients, many of whom may be asymptomatic carriers of SARS-CoV-2. Meticulous utilization of personal protective equipment (PPE) by ophthalmologists was recently emphasized in updates published by the American Academy of Ophthalmology [6], and in 
other publications as well [7]. However, practical implementation of these guidelines has been challenging due to a lack of PPE and economical and administrative contingencies at the local level.

The first confirmed case of COVID-19 in Israel was reported on February 21, 2020 [8]. Only several weeks later, with over 300 confirmed cases, ophthalmologic clinical and surgical practices were in the process of being curtailed.

In the midst of the uncertainty, we sought to assess the preparedness of local practicing ophthalmologists in Israel to confront the viral outbreak by collecting their recent experiences and perceptions regarding their practice during the unraveling pandemic.

\section{Methods}

\section{Data collection}

One hundred and sixty-seven ophthalmologists practicing in Israel from various regions and clinical setups completed a 17-item online anonymous questionnaire using SurveyMonkey.com. This application is a secure platform for building and managing online surveys (Appendix 1). Survey invitations were distributed to ophthalmologists through email and the WhatsApp messenger application. The questionnaire was published online on March 17, 2020, was available online for $72 \mathrm{~h}$, and comprised objective multiple-choice questions and two open questions. At the time of survey distribution, no COVID-19-related guidelines had been published for ophthalmology departments and clinics. Several hours after the survey was initiated the Israeli Health Ministry announced its intention to cease all nonurgent ambulatory services and elective surgeries beginning March 22. Data collection included demographic properties, clinical practice attributes, subspecialty, and PPE utilization during the COVID-19 outbreak. The study was approved by the institutional ethics committee of Shaare Zedek Medical Center, Jerusalem.

\section{Statistical analysis}

All variables were categorical, and as such, they were summarized by frequency counts and percentages. In order to test the association between two variables, the Chi-squared test was performed, considering a $P$ value of $<0.05$ to be statistically significant. Analyses were performed in the SPSS 25.0 (SPSS Inc., Chicago, IL).

\section{Results}

\section{Demography, subspecialties, and clinical setups}

Demography and clinical and surgical volume of survey participants are detailed in Table 1.

According to the Israel Ophthalmological Society, there are approximately 660 practicing ophthalmologists. One hundred and sixty-seven $(25.3 \%)$ of these physicians responded to this survey. Ninety-nine (59.3\%) respondents were men and 68 (40.7\%) were women. Sixty-three $(37.7 \%)$ respondents were under the age of 40 , seventy-two (43.1\%) were between the ages of 40-60, and thirty-two (19.2\%) exceeded 60 years of age.

One hundred and eleven respondents (66.5\%) identified their primary workplace as a hospital, $34(20.3 \%)$ worked in outpatient clinics, and 22 (13.2\%) physicians worked in private practice. Forty-nine $(29.3 \%)$ participants identified as general ophthalmologists $94(56.3 \%)$ practiced in multiple subspecialties and 24 (14.4\%) identified as ophthalmology residents.

Ninety-six participants $(57.4 \%)$ provided identification for their place of work. Of these, $53.1 \%$ were located in Central Israel, $24.0 \%$ were located in Northern Israel, and $13.5 \%$ were located in Southern Israel. This provides a representative sample of Israeli ophthalmologists.

Most respondents (38.9\%) stated that they treat more than 75 patients per week, $28.7 \%$ treat between 51 and 75 patients per week, $20.4 \%$ treat between 26 and 50 patients per week, and $12 \%$ treat up to 25 patients per week.

Most respondents (78.7\%) reported operating on as many as 10 patients a week.

\section{Guideline awareness and PPE utilization}

Responses regarding clinical setting adjustments, guideline awareness, and PPE utilization are summarized in Table 2.

Eighty-eight ophthalmologists $(52.7 \%)$, most of whom practice in hospitals $(61.2 \%)$, stated that no active measures were taken to decrease the number of patients seen in clinics since the beginning of the COVID-19 outbreak until March 20th when the survey 
Table 1 Demographics and patient volumes of 167 survey participants
Table 2 Clinical setting adjustments, guidelines awareness and PPE utilization

\begin{tabular}{lcccr}
\hline Variables & Hospital & Community clinic & Private clinic & Total, $n(\%)$ \\
\hline Age (years) & & & & \\
$<40, n(\%)$ & $54(48.6)$ & $6(17.6)$ & $3(13.6)$ & $63(37.7)$ \\
$40-60, n(\%)$ & $49(44.1)$ & $17(50.0)$ & $6(27.3)$ & $72(43.1)$ \\
$>60, n(\%)$ & $8(7.3)$ & $11(32.4)$ & $59.1(13)$ & $32(19.2)$ \\
Gender & & & & \\
Male, $n(\%)$ & $70(63.1)$ & $15(44.1)$ & $14(63.6)$ & $99(59.3)$ \\
Female, $n(\%)$ & $41(36.9)$ & $19(55.9)$ & $6(36.7)$ & $68(40.7)$ \\
Clinic patients $(/$ week) & & & & \\
$\leq 25, n(\%)$ & $15(13.5)$ & $3(8.8)$ & $3(13.7)$ & $34(20.4)$ \\
$26-50, n(\%)$ & $28(25.2)$ & $3(8.8)$ & $5(22.7)$ & $48(28.7)$ \\
$51-75, n(\%)$ & $36(32.5)$ & $7(20.6)$ & $12(54.5)$ & $65(38.9)$ \\
$>75, n(\%)$ & $32(28.8)$ & $21(61.8)$ & & $118(78.7)$ \\
Surgeries*(/week) & & & $13(81.2)$ & $28(18.7)$ \\
$\leq 10, n(\%)$ & $84(77.1)$ & $21(84.0)$ & $2(12.5)$ & $1(0.6)$ \\
$11-20, n(\%)$ & $23(21.1)$ & $3(12.0)$ & $0(0)$ & $3(2.0)$ \\
$21-30, n(\%)$ & $1(0.9)$ & $0(0)$ & $22(13.2)$ & $167(100)$ \\
$>30, n(\%)$ & $1(0.9)$ & $1(4.0)$ & $34(20.3)$ &
\end{tabular}

Data is presented in frequency counts and percentages, by categories of clinical setups

* This question was skipped by 17 respondents

\begin{tabular}{|c|c|c|c|c|}
\hline & Hospital & Community clinic & Private clinic & Total \\
\hline \multicolumn{5}{|c|}{ Have any active measures been taken to decrease the number of patients? } \\
\hline \multicolumn{5}{|l|}{ Outpatients } \\
\hline Yes, $n(\%)$ & $43(38.7)$ & $20(58.8)$ & $16(72.7)$ & $79(47.3)$ \\
\hline No, $n(\%)$ & $68(61.3)$ & $14(41.2)$ & $6(27.3)$ & $88(52.7)$ \\
\hline \multicolumn{5}{|l|}{ Surgeries } \\
\hline Yes, $n(\%)$ & $25(22.7)$ & $13(48.1)$ & $8(50.0)$ & $46(30.1)$ \\
\hline No, $n(\%)$ & $85(77.3)$ & $14(51.9)$ & $8(50.0)$ & $107(69.9)$ \\
\hline \multicolumn{5}{|c|}{ Triage questionnaire on admission? } \\
\hline Yes, $n(\%)$ & $52(46.9)$ & $14(42.4)$ & $11(50.0)$ & $77(46.4)$ \\
\hline No, $n(\%)$ & $59(53.1)$ & $19(57.6)$ & $11(50.0)$ & $89(53.6)$ \\
\hline \multicolumn{5}{|c|}{ Were you informed of COVID-19 guidelines? } \\
\hline Yes, $n(\%)$ & $76(68.5)$ & $17(50.0)$ & $8(36.4)$ & $101(60.5)$ \\
\hline No, $n(\%)$ & $35(31.5)$ & $17(50.0)$ & $14(63.6)$ & $66(39.5)^{* *}$ \\
\hline \multicolumn{5}{|c|}{ Were you provided with the PPE you use? } \\
\hline Yes, $n(\%)$ & $67(60.4)$ & $17(51.5)$ & $0(0)$ & $84(50.6)$ \\
\hline No, $n(\%)$ & $44(39.6)$ & $16(48.5)$ & $22(100.0)$ & $82(49.4)^{* * *}$ \\
\hline
\end{tabular}

Data is presented in frequency counts and percentages, by categories of clinical setups. Responses were analyzed using Chi-squared tests. ${ }^{* *} P<0.01,{ }^{* * *} P<0.001$ 
Table 3 Perceptions regarding ophthalmology services during the COVID-19 outbreak

\begin{tabular}{lcccr}
\hline & Hospital & Community clinic & Private clinic & Total \\
\hline \multicolumn{4}{l}{ What level of clinic services should we be providing? } \\
$\begin{array}{l}\text { Full, } n(\%) \\
\text { Partial, } n(\%)\end{array}$ & $5(4.5)$ & $1(3.0)$ & $2(9.1)$ & $8(4.8)$ \\
Emergency only, $n(\%)$ & $75(67.6)$ & $18(52.9)$ & $8(36.4)$ & $54(32.3)$ \\
What level of surgical service should we be providing? & $12(54.5)$ & $105(62.9)$ \\
Full, $n(\%)$ & $4(3.6)$ & $0(0)$ & $1(4.8)$ & $5(3.0)$ \\
Partial, $n(\%)$ & $27(24.3)$ & $7(21.2)$ & $6(28.6)$ & $40(24.3)$ \\
Emergency only, $n(\%)$ & $80(72.1)$ & $26(78.8)$ & $14(66.6)$ & $120(72.7)$ \\
\hline
\end{tabular}

Data is presented in frequency counts and percentages, by categories of clinical setups. Responses were analyzed using Chi-squared tests was completed. A similar trend of routine practice of surgical services was observed $(69.9 \%$ of all respondents, $77.2 \%$ of whom reported working in a hospital). At the time of the survey, most respondents $(n=89$, $53.6 \%$ ) reported that no COVID-19 triage questionnaire had been provided to patients. Most participants $(n=$ $101,60.5 \%$ ) were informed by their superiors of official COVID-19 guidelines for ophthalmologists. However, exclusion of hospital practitioners reversed the trend, as the majority of community and private ophthalmologists $(n=31,55.3 \%)$ were not aware of such guidelines $(P=0.006)$. The most commonly utilized PPE was surgical face masks $(n=135,80.8 \%)$ and protective slitlamp shields $(n=114,68.3 \%)$. Approximately, half of the respondents $(n=82,49.4 \%)$ reported providing PPE for themselves. When private practitioners were excluded, this portion of participants decreased but was still evident $(n=60,41.7 \%, P<0.001)$. A majority of ophthalmologists $(n=121,72.9 \%)$ mentioned the slitlamp examination as procedure during which they felt the most exposed to infection, followed by the instillation of eye-drops $(n=28,16.9 \%)$.

\section{Perceptions regarding ophthalmology services during the COVID-19 outbreak}

Most participants $(62.9 \%)$ were of the opinion that practice should be limited to emergency services (Table 3.).

This trend remained consistent in all clinical setups $(67.6 \%$, $52.9 \%$, and $54.5 \%$ for hospital, community clinics, and private practice, respectively, $P=0.344)$. Similarly, the majority of respondents $(n=120,72.7 \%)$ noted that surgical activity should be limited to emergencies. This remained consistent in all clinical locations $(72.1 \%, 78.8 \%$, and $66.7 \%$ for hospital, community clinics, and private practice, respectively, $P=0.779)$.

\section{Discussion}

The risk of procedural viral transmission in ophthalmology was clearly demonstrated in reports from Hong Kong during the SARS coronavirus (SARS-CoV) outbreak in the years 2002-2003 [9]. Furthermore, recent publications regarding the present COVID-19 pandemic have demonstrated a high level of transmissibility in routine ophthalmologic practice [10]. In this surveybased study, we obtained the opinions of Israeli ophthalmologists regarding the risk of ophthalmologic practice during the local early stages of the COVID-19 outbreak. Responses were received from ophthalmologists in many departments in Israeli hospitals as well as health maintenance organizations and private clinics providing a highly representative sample. At the time of the survey, 3 weeks after the first reported case of COVID-19 in Israel, most ophthalmologists reported starting to utilize PPE during routine clinic visits. Many reported the need to obtain PPE independently, including ophthalmologists working in hospital settings. A large number of practitioners described not being informed of official COVID-19 clinic guidelines, especially ophthalmologists practicing in busy outpatient clinics of health maintenance organizations (Kupat Holim). The majority of respondents were of the opinion that clinic and surgical services should have been reduced to emergency services only. At the time of the survey while the outbreak was progressing, most physicians reported no reduction of patient volume. 
The lack of both guideline distribution and a decision to curtail ambulatory services during the early days of the outbreak may have contributed to viral spread. Timely implementation of guidelines during this new pandemic is challenging for the health system. Delay of action, however, may endanger both the medical staff and patients at critical moments. This is particularly consequential in an ophthalmology setting where close contact occurs between elderly and asymptomatic young individuals recently identified as active viral carriers $[11,12]$.

Since the distribution of this survey, Israeli guidelines have been disseminated regarding proper PPE and reductions of ambulatory services in ophthalmology practice. These guidelines were published on March 23, 2020, by the Israeli Ophthalmological Society and are based on recommendations of the American Academy of Ophthalmology [6, 13]. Protective measures for physicians are based on the level of suspicion for COVID-19 infection. Clinical and surgical services are limited to urgent cases only, and the utilization of a slit-lamp breath shield is recommended.

Although the guidelines have been published, the availability of proper protective equipment is often lacking. As of the end of March, the international pandemic has progressed, and more than sixty physicians in Italy have already died, and many others in Europe and in the USA have tested positive for the virus [14]. The best explanation to why otherwise healthy individuals would succumb to the infection is related to the extent of the viral load on exposure and the mass of the COVID-19 inoculum [15]. This can be greatly reduced by utilization of proper PPE [16], specifically N-95 masks, while performing invasive ophthalmologic procedures and the greater use of COVID-19 testing to identify patients with the viral infection. A promising advance is the use of antibody testing to identify physicians and healthcare workers who possess immunity to the virus, enabling these individuals to treat patients while ensuring their own safety [17]. As of April 6th, for the Israeli population estimated at 8,972,000 as of 2019, there are presently 8904 individuals who have tested positive for COVID-19, 56 of whom have died [18]. To our knowledge, there is one ophthalmologist who has tested positive for COVID 19. Routine testing of physicians is not presently being provided.

There are several limitations to this study. Firstly, due to the rapid unfolding of events related to an increasing number of individuals with COVID-19 infection, several hours after the survey was released, the Israeli Health Ministry announced its intention to cease all non-urgent ambulatory services and surgeries beginning March 22. Previous to this, no restriction of ambulatory services had been considered. Secondly, the number of respondents was limited to $\sim 25 \%$ of practicing ophthalmologists due to the need to limit the access of the survey to $72 \mathrm{~h}$ to reflect the changing healthcare environment.

\section{Conclusion}

As the COVID-19 viral outbreak rapidly progressed and individuals sequestered themselves in their homes, physicians and other healthcare workers were called upon to treat patients in a higher-risk reality. The findings from this questionnaire-based study show that at the onset of the pandemic in Israel, ophthalmologists described themselves as inadequately protected from COVID 19 infection and felt a lack of support. It is notable that the COVID-19 outbreak in Wuhan, China, was initially identified by an ophthalmologist, Dr. Li Wenliang, who at 33 years of age contracted the virus in the early days of the outbreak and recently died of the disease [19]. Both his story and this survey emphasize the importance of attending to the safety of healthcare workers. For the first time in the lives of most physicians, the routine care of patients becomes a personal health care risk. As a result, physicians must prioritize their own health and safety as they continue to care for their patients.

Authors' contributions LMW, EA, MT, YW, and DZ wrote the manuscript, provided data for the tables, and conducted the survey. MB-C provided statistical analyses. All authors reviewed the final manuscript.

\section{Compliance with ethical standards}

Conflict of interest The authors declare that they have no conflict of interest.

Ethical approval All procedures performed in studies involving human participants were in accordance with the ethical standards of the ethics committee of Shaare Zedek Medical Center, Jerusalem, and with the 1964 Helsinki declaration and its later amendments or comparable ethical standards.

The survey was approved by the committee and was sent to Israeli ophthalmologists. The decision of the Shaare Zedek Medical Center Helsinki Committee in a letter dated 18 March 2020 reflecting previous verbal conversations was that the response to the survey by the Israeli ophthalmologists was an implied written consent to participate in the study.

This study was performed in line with the principles of the Declaration of Helsinki. Approval was granted by the Ethics Committee of Shaare Zedek Medical Center, Jerusalem.

Informed consent Informed consent was deemed to be implied by survey participations as per the Shaare Zedek Medical Center Helsinki Committee as explained previously. 


\section{Appendix 1. Ophthalmology Practice in Israel during the COVID-19 Pandemic}

1. What is your clinical setup? If you practice in more than one setting, please refer to the primary setting:

- Hospital

- Community clinic

- Private practice

2. What is your sub-specialty?

- General ophthalmology

- Cornea

- Glaucoma

- Cataract

- Retina

- Neuro-ophthalmology

- Pediatric ophthalmology

- Oculoplastics

- Ocular oncology

- Uveitis

- Ophthalmologist in training (Resident)

3. What is your age?

- Under 40

- 40-60

- Over 60

4. What is your gender?

- Female

- Male

5. Approximately how many patients do you see in an outpatient setting per week?

- Up to 25 patients

- 26-50 patients

○ 51-75 patients

- More than 75 patients

6. Approximately how many patients do you operate on per week?

- Up to 10

- 11-20

○ $21-30$

○ More than 30

7. Since the COVID-19 outbreak (over the last few weeks) have any active measures been taken to decrease the number of patients that attend your clinic?

- Yes

$\circ \quad$ No

8. Since the COVID-19 outbreak (over the last few weeks) have there been any active measures taken to decrease the number of patients that you operate on?

- Yes

$\circ$ No 
9. Which protective measures are you currently utilizing when examining clinic patients? (Multiple answers may be checked)

○ Mask

- Eye protection (goggles or eye shield)

- Gown

- Protective slit lamp shield

- None of the above

10. Who provided you with these protective measures?

$\circ$ They were provided for me

$\circ$ I provided them for myself

11. In your opinion, in which of the following actions are you most exposed to infection?

$\circ$ Instilling eye drops

- Slit-lamp examination

- Indirect ophthalmoscopy

- Consultations in wards

12. In your opinion, given the current status of COVID-19 in Israel, what level of clinic services should we be providing our patients with?

- Full ambulatory service

- Partial ambulatory service

$\circ$ Emergency services only

13. In your opinion, given the current status of COVID-19 in Israel, what level of surgical service should we be providing our patients with?

- Full surgery schedule

- Partial surgery schedule

$\circ$ Emergency surgeries only

14. Were you informed by your superiors of any official COVID-19 guidelines and updates for ophthalmologists?

- Yes

$\circ$ No

15. In your clinical setup are your patients subject to a COVID-19 questionnaire on admission?

- Yes

$\circ$ No

16. For future follow-up purposes, and only if you are willing to share, please state your place/s of work (name of institution and city

17. Please feel free to leave any other comments or suggestions: 


\section{References}

1. WHO Director-General's opening remarks at the media briefing on COVID-19 - 16 March 2020. Who.int. https://www.who.int/dg/ speeches/detail/who-director-general-s-opening-remarks-at-themedia-briefing-on-covid-19\%2D\%2D-16-march-2020. Published 2020. Accessed 30 Mar 2020

2. COVID-19. European Centre for Disease Prevention and Control. https://www.ecdc.europa.eu/en/novel-coronavirus-china. Published 2020. Accessed 30 Mar 2020

3. Coronavirus Disease 2019 (COVID-19) - Symptoms. Centers for Disease Control and Prevention. https://www.cdc.gov/coronavirus/ 2019-ncov/symptoms-testing/symptoms.html. Published 2020. Accessed 30 Mar 2020

4. COVID-19 reports. Imperial College London. https://www. imperial.ac.uk/mrc-global-infectious-disease-analysis/news $\% 2 \mathrm{D} \%$ 2Dwuhan-coronavirus. Published 2020. Accessed 30 Mar 2020

5. Chang D, Xu H, Rebaza A, Sharma L, Dela Cruz C (2020) Protecting health-care workers from subclinical coronavirus infection. Lancet Respir Med 8:e13. https://doi.org/10.1016/s22132600(20)30066-7

6. Important coronavirus updates for ophthalmologists. American Academy of Ophthalmology. https://www.aao.org/headline/alertimportant-coronavirus-context. Published 2020. Accessed 30 Mar 2020

7. Li J, Lam D, Chen Y, Ting D (2020) Novel coronavirus disease 2019 (COVID-19): the importance of recognizing possible early ocular manifestation and using protective eyewear. $\mathrm{Br} \mathrm{J}$ Ophthalmol 104:297-298. https://doi.org/10.1136/bjophthalmol2020-315994

8. Israel confirms first coronavirus case as cruise ship returnee diagnosed. Timesofisrael.com. https://www.timesofisrael.com/israelconfirms-first-coronavirus-case-as-cruise-ship-returneediagnosed/. Published 2020. Accessed 30 Mar 2020

9. Lai J (2006) Precautions in ophthalmic practice in a hospital with a major acute SARS outbreak: an experience from Hong Kong. Eye. 21:304-305. https://doi.org/10.1038/sj.eye.6702579
10. Lu C, Liu X, Jia Z (2020) 2019-nCoV transmission through the ocular surface must not be ignored. Lancet 395:e39. https://doi. org/10.1016/s0140-6736(20)30313-5

11. Bai Y, Yao L, Wei T et al (2020) Presumed asymptomatic carrier transmission of COVID-19. JAMA. https://doi.org/10.1001/jama. 2020.2565

12. Li R, Pei S, Chen B et al (2020) Substantial undocumented infection facilitates the rapid dissemination of novel coronavirus (SARSCoV2). Science. https://doi.org/10.1126/science.abb3221

13. New recommendations for urgent and nonurgent patient care American Academy of Ophthalmology. https:/www.aao.org/ headline/new-recommendations-urgent-nonurgent-patient-care. Accessed 6 Apr 2020

14. More than 60 doctors in Italy have died in COVID-19 pandemic. Medscape. https://www.medscape.com/viewarticle/927753. Published 2020. Accessed 31 Mar 2020

15. Topol: US betrays healthcare workers in coronavirus disaster. Medscape. https://www.medscape.com/viewarticle/927811. Published 2020. Accessed 31 Mar 2020

16. Lai T, Tang E, Chau S, Fung K, Li K (2020) Stepping up infection control measures in ophthalmology during the novel coronavirus outbreak: an experience from Hong Kong. Graefes Arch Clin Exp Ophthalmol. https://doi.org/10.1007/s00417-020-04641-8

17. Amanat F, Nguyen T, Chromikova V et al. (2020) A serological assay to detect SARS-CoV-2 seroconversion in humans. Medrxiv. https://doi.org/10.1101/2020.03.17.20037713

18. Coronavirus: 57 dead, 8,904 known infected. Israelnationalnews. com. http://www.israelnationalnews.com/News/News.aspx/ 278388/. Published 2020. Accessed 6 Apr 2020

19. Petersen E, Hui D, Hamer D et al (2020) Li Wenliang, a face to the frontline healthcare worker. The first doctor to notify the emergence of the SARS-CoV-2, (COVID-19), outbreak. Int J Infect Dis 93: 205-207. https://doi.org/10.1016/j.ijid.2020.02.052

Publisher's note Springer Nature remains neutral with regard to jurisdictional claims in published maps and institutional affiliations. 